\title{
Some properties of implications via aggregation functions and overlap functions
}

\author{
H. Zapata ${ }^{1}$, H. Bustince², L. De Miguel², C. Guerra² . \\ ${ }^{1}$ Universidad Central de Venezuela, \\ Venezuela \\ E-mail: hugozapata1@hotmail.com \\ ${ }^{2}$ Departamento de Automatica y Computacion, Universidad Publica de Navarra, \\ Campus Arrosadia, 31006, Pamplona, Navarra, Spain.
}

E-mail: bustince@unavarra.es, carlos.guerra@unavarra.es, laura.demiguel@unavarra.es

Received 15 October 2013

Accepted 19 May 2014

\begin{abstract}
In this work, using the identification between implication operators and aggregation functions, we study the implication operators that are recovered from overlap functions. In particular, we focus in which properties of implication operators are preserved. We also study how negations can be defined in terms of overlap functions.
\end{abstract}

Keywords: Implication operator, aggregation function, negation, overlap function.

\section{Introduction}

The concept of implication operator was introduced in 1982 by Dombi ${ }^{15}$. It arises from the minimal set of properties that the operators usually used in aggregation or data fusion procedures must fulfill.

From the works of Bandler and Kohout ${ }^{3}$ in 1980 and of Trillas and Valverde ${ }^{24}$ in 1981, Klir and Folger in $1988^{19}$ settle that the logic operation of implication is as essential for approximate reasoning as it is for reasoning within classical two-valued logic. In general, a fuzzy implication $I$ is a function $I:[0,1]^{2} \rightarrow[0,1]$ such that for every two possible truth values $a$ and $b$ of propositions $p$ and $q$ respectively, $I(a, b)$ represents the truth value of the conditional proposition if $p$, then $q$.

Implication operators have been widely studied and used in different fields $s^{1,2,20}$. For instance, in image processing they have been used to build mea- sures for image comparison ${ }^{6,8}$ when we are working with algorithms which allow us to extract objects from the considered image ${ }^{7}$. In order to use such algorithms in an image composed of a single object and the background, the first thing to do is to represent both the object and the background by means of a fuzzy set.

Moreover, it is well-known that implication operators can be recovered from aggregation functions and vice-versa ${ }^{14}$. In particular, certain properties of implication operators can be directly translated into the aggregation function setting, and properties of aggregation functions admit a straight interpretation in terms of implication operators.

It has been proved that in multiclass problems the difficulty in providing an optimal solution is directly proportional to the overlapping that exists between the considered classes. This fact, together with the idea of consistency index for fuzzy rules given by 
Zadeh $^{26}$, led to the introduction of the concept of overlap function ${ }^{10}$. Overlap functions are mappings $[0,1]^{2} \rightarrow[0,1]$ that measure the degree of overlapping between the membership degree of an element to two fuzzy sets. Moreover, duality allows to define also the concept of grouping function ${ }^{13}$ in such a way that some of the most relevant properties demanded to triplets in terms of $\mathrm{t}$-norms and $\mathrm{t}$-conorms are preserved. However, neither overlap nor grouping functions need to be associative, which is an advantage in some fields of application where associativity is not a natural property to be demanded.

Since overlap (and grouping) functions are particular instances of aggregation functions, in this work we aim at the following objectives:

1. To study how negations can be recovered from overlap functions.

2. To analyze the properties of implication operators obtained from overlap functions.

This paper is organized as follows. After recalling some preliminaries notions in Section 2, we study in Section 3 those negations constructed from overlap functions. In Section 4, the relationship between implication operators and overlap functions is investigated. We finish in Section 5 with some conclusions and acknowledgments.

\section{Preliminaries}

In this section we recall some well-known concepts in order to fix the notations used further on. A reference for these concepts is ${ }^{17}$.

Definition 1. An automorphism on the unit interval is an increasing, continuous bijection $\varphi:[0,1] \rightarrow$ $[0,1]$.

Definition 2. A negation is a decreasing function $N:[0,1] \rightarrow[0,1]$ such that $N(0)=1$ and $N(1)=0$. A negation is called:

- frontier if $N(x) \in\{0,1\}$ if and only if $x \in\{0,1\}$.

- strict if $N$ is continuous and strictly decreasing.

- strong if $N$ is involutive, that is, $N(N(x))=x$ for all $x \in[0,1]$.
Example 1. The least negation $N_{*}$ is defined by

$$
N_{*}(x)= \begin{cases}1 & \text { if } x=0 \\ 0 & \text { otherwise }\end{cases}
$$

The greatest negation $N^{*}$ is given by

$$
N^{*}(x)=\left\{\begin{array}{lc}
0 & \text { if } x=1 \\
1 & \text { otherwise }
\end{array}\right.
$$

The most used negation is the standard (or Zadeh's) negation $N_{Z}(x)=1-x$. Notice that $N_{Z}$ is strong. The following characterization of strong negations was proven by Trillas.

Theorem 1. ${ }^{23}$ A function $N:[0,1] \rightarrow[0,1]$ is a strong negation if and only if there exists an automorphism $\varphi:[0,1] \rightarrow[0,1]$ such that

$$
N(x)=\varphi^{-1}(1-\varphi(x))
$$

for all $x \in[0,1]$.

That is, a strong negation can be seen as a perturbation, via an automorphism $\varphi$, of Zadeh's negation.

Definition 3. An ( $n$-ary) aggregation function is an increasing mapping $A:[0,1]^{n} \rightarrow[0,1]$ such that $A(0, \ldots, 0)=0$ and $A(1, \ldots, 1)=1$.

In the present work, we mainly deal with binary $(n=2)$ aggregation functions.

\section{Example 2.}

- The greatest aggregation function $A^{*}$ is defined by

$$
A^{*}(x, y)= \begin{cases}0 & \text { if } x=0 \text { and } y=0 \\ 1 & \text { otherwise. }\end{cases}
$$

- The least aggregation function $A_{*}$ is defined by

$$
A_{*}(x, y)= \begin{cases}1 & \text { if } x=1 \text { and } y=1 \\ 0 & \text { otherwise }\end{cases}
$$

Given a mapping $\varphi:[0,1] \rightarrow[0,1]$ and an aggregation function $A:[0,1]^{2} \rightarrow[0,1]$, we denote by $A_{\varphi}$ the mapping defined by

$$
A_{\varphi}(x, y)=A(\varphi(x), \varphi(y)) .
$$


If $\varphi$ is increasing, $\varphi(0)=0$ and $\varphi(1)=1$, then $A_{\varphi}$ is also an aggregation function.

Definition 4. An aggregation function $A$ is called associative if the identity

$$
A(A(x, y), z)=A(x, A(y, z))
$$

holds for all $x, y, z \in[0,1]$.

Definition 5. Let $A$ be a binary aggregation function.

(i) An element $e_{A} \in[0,1]$ is said to be a neutral element for $A$ if $A\left(x, e_{A}\right)=A\left(e_{A}, x\right)=x$ for all $x \in[0,1]$.

(ii) An element $\left.\left.x_{0} \in\right] 0,1\right]$ is called a zero-divisor for $A$ if there exists $x \in] 0,1]$ such that $A\left(x, x_{0}\right)=$ $A\left(x_{0}, x\right)=0$.

(iii) An element $a \in[0,1]$ is said to be an annihilator for $A$ if $A(a, x)=A(x, a)=a$ for all $x \in[0,1]$.

Several studies have focused on specific classes of aggregation operators, from both a theoretical and a practical point of view. Two of the classes receiving more attention are those of t-norms and overlap functions.

Definition 6. A t-norm is an associative symmetric aggregation function $T:[0,1]^{2} \rightarrow[0,1]$ such that $T(x, 1)=(1, x)=x$ for all $x \in[0,1]$ (that is, 1 is a neutral element for $T$ ).

Definition 7. An overlap function is a function $G_{O}:[0,1]^{2} \rightarrow[0,1]$ such that:

(O1) $G_{O}$ is symmetric;

(O2) $G_{O}(x, y)=0$ if and only if $x y=0$;

(O3) $G_{O}(x, y)=1$ if and only if $x y=1$;

$(O 4) G_{O}$ is increasing;

(O5) $G_{O}$ is continuous. in $^{10}$.

A deep study on overlap functions can be found

\section{Example 3.}

1. Any continuous t-norm without divisors of zero is an example of overlap function. In particular, both $G_{O}(x, y)=\min (x, y)$ and $G_{O}(x, y)=x y$ are t-norms and consequently overlap functions.
2. The function $G_{O}(x, y)=(x y)^{p}$ for $p>0$ is an overlap function. Notice that for $p \neq 1 G_{O}$ is not associative.

3. The function $G_{O}(x, y)=\min \left(x^{p}, y^{p}\right)^{q}$ for $p, q>0$ is an overlap function. Notice that for $p, q<1, G_{O}(x, y) \geqslant \min (x, y)$.

Another key concept for our work is that of implication operator ${ }^{3,24}$. We start recalling its definition (in the sense of Fodor ${ }^{16}$ ) and the main properties that can be demanded to such an operator.

Definition 8. An implication operator is a function $I:[0,1]^{2} \rightarrow[0,1]$ such that

$(F A)$ If $x \leqslant z$ then $I(x, y) \geqslant I(z, y)$ for all $y \in[0,1]$ (decreasing in the first variable).

(SI) If $y \leqslant z$ then $I(x, y) \leqslant I(x, z)$ for all $x \in[0,1]$ (increasing in the second variable).

(DF) $I(0, x)=1$ for all $x \in[0,1]$ (dominance of falsity).

(DT) $I(x, 1)=1$ for all $x \in[0,1]$ (dominance of truth of consequent).

(BC) $I(1,0)=0$ (the boundary condition).

Definition 8 recalls the minimal properties of implication operators. Nevertheless, their study has led to several other properties which further characterize their behaviour.

Definition 9. ${ }^{1,4}$ The following properties can be used to outline the behaviour of an implication operator $I$.

(NP) $I(1, x)=x$ for all $x \in[0,1]$ (neutrality of truth).

(EP) $I(x, I(y, z))=I(y, I(x, z))$ for all $x, y, z \in[0,1]$ (exchange principle).

$(O P) I(x, y)=1$ if and only if $x \leqslant y$ (ordering property).

$(S N) N(x)=I(x, 0)$ is a strong negation.

(IP) $I(x, x)=1$ for all $x \in[0,1]$ (identity principle).

(CP) $I(x, y)=I(N(y), N(x))$ for some strong negation $N$ and for all $x, y \in[0,1]$ (contrapositive property).

(IA) $I(x, y) \geqslant y$ for all $x, y \in[0,1]$.

(IB) $I$ is continuous. 
(IC) $I(x, N(x))=N(x)$ for some strong negation $N$ and for all $x \in[0,1]$.

Apart from the properties in the previous definition, two other conditions are of great relevance for specific applications ${ }^{12}$.

(P1) $I(x, y)=1$ if and only if $x=0$ or $y=1$.

(P2) $I(x, y)=0$ if and only if $x=1$ and $y=0$.

\section{Overlap functions and negations}

In this section we stablish the relationship of the concepts of overlap functions and negations.

Proposition 2. Let $F:[0,1] \rightarrow[0,1]$ be a function. Then the following statements are equivalent.

(a.) F is a negation.

(b.) For every overlap function $G_{O}:[0,1]^{2} \rightarrow[0,1]$ the function

$$
N_{G_{O}}(x)=G_{O}(1, F(x))
$$

is a negation.

Proof. If $F$ is a negation and $G_{O}:[0,1]^{2} \rightarrow[0,1]$ is an overlap function, then $N_{G_{O}}$ is decreasing. Moreover

$$
N_{G_{O}}(0)=G_{O}(1, F(0))=G_{O}(1,1)=1
$$

and

$$
N_{G_{O}}(1)=G_{O}(1, F(1))=G_{O}(1,0)=0,
$$

so $N_{G_{O}}$ is a negation.

Let us assume statement (b.) holds for every overlap function $G_{O}$. If $G_{O}$ is an overlap function with neutral element 1 , then

$$
N_{G_{O}}(x)=G_{O}(1, F(x))=F(x),
$$

and since $N_{G_{O}}$ is a negation, the result follows.

Corollary 3. Let $F:[0,1] \rightarrow[0,1]$ be a function. Then the following statements are equivalent.

(a.) F is a frontier negation. (b.) For every overlap function $G_{O}, N_{G_{O}}$ defined as in Proposition 2 is a frontier negation.

Proof. It is straight from the boundary conditions in the definition of overlap function.

Example 4. Let be $p>0$ and $G_{O}(x, y)=(x y)^{p}$.

1. If $F=N_{*}$

$$
N_{G_{O}}(x)=G_{O}\left(1, N_{*}(x)\right)= \begin{cases}1 & \text { if } x=0 \\ 0 & \text { otherwise }\end{cases}
$$

and we recover $N_{*}$.

2. If $F=N^{*}$

$$
N_{G_{O}}(x)=G_{O}\left(1, N^{*}(x)\right)= \begin{cases}0 & \text { if } x=1 \\ 1 & \text { otherwise }\end{cases}
$$

and we recover $N^{*}$.

3. However, if we consider $F(x)=1-x^{q}$ for some $q>0$, we see that

$$
N(x)=G_{O}(1, F(x))=\left(1-x^{q}\right)^{p}
$$

which is different from $F$ as long as $p \neq 1$.

From the previous results we characterize negations through aggregation functions that satisfy suitable boundary conditions. We can also recover any negation from these aggregation functions using one fixed strong negation.

Lemma 4. Let $\varphi:[0,1] \rightarrow[0,1]$ be an increasing continuous function such that $\varphi(x)=0$ if and only if $x=0$ and $\varphi(x)=1$ if and only if $x=1$. The function $G$, given by

$$
G(x, y)=\varphi(x) \varphi(y)
$$

is an overlap function.

Proof. Straight.

Remark 1. Note that, in fact, the same result holds if we define

$$
G(x, y)=G_{O}(\varphi(x), \varphi(y))
$$

for some overlap function $G_{O}$.

Then we can state the main result.

Theorem 5. Let $N_{0}$ be a strict negation and $F$ : $[0,1] \rightarrow[0,1]$ a mapping. Then the following statements are equivalent. 
(a.) F is a continuous frontier negation.

(b.) There exists an overlap function $G_{O}:[0,1]^{2} \rightarrow$ $[0,1]$ such that

$$
F(x)=G_{O}\left(1, N_{0}(x)\right)
$$

for all $x \in[0,1]$. Moreover, this overlap function is given by

$$
G_{O}(x, y)=F\left(N_{0}^{-1}(x)\right) F\left(N_{0}^{-1}(y)\right) .
$$

Proof. The fact that statement $(b$.) implies statement (a.) follows from Corollary 3 because $G_{O}$ is continuous and every strict negation is a frontier negation.

If $(a$.) holds, and $F$ is a continuous frontier negation, we define

$$
\varphi(x)=F\left(N_{0}^{-1}(x)\right),
$$

which satisfies the properties in the statement of Lemma 4. If

$$
G_{O}(x, y)=\varphi(x) \varphi(y)
$$

then we have that

$$
N_{G_{O}}(x)=G_{O}\left(1, N_{0}(x)\right)=F\left(N_{0}^{-1}\left(N_{0}(x)\right)\right)=F(x)
$$

and the result follows.

In particular, if we fix $N_{0}$ as the standard negation, namely, $N_{0}=N_{Z}$, we arrive at the following result.

Corollary 6. A function $F:[0,1] \rightarrow[0,1]$ is a continuous frontier negation if and only if there exists an overlap function $G_{O}:[0,1]^{2} \rightarrow[0,1]$ such that $F(x)=G_{O}(1,1-x)$ for all $x \in[0,1]$. This overlap function is given by $G_{O}(x, y)=F(1-x) F(1-y)$. Proof. Straight.

Notice that, in general, the overlap function $G_{O}$ needs not be unique. For instance, observe that the standard negation $N(x)=1-x$ can be recovered by any overlap function which has neutral element 1 .

\section{Implication operators and overlap functions}

For the development of this section the following result is crucial.
Proposition 7. ${ }^{14}$ Let $N$ be a negation and $A$ a binary aggregation function such that $A(0,1)=A(1,0)=0$. Then, the function $I_{A}:[0,1]^{2} \rightarrow[0,1]$ given by

$$
I_{A}(x, y)=N(A(x, N(y)))
$$

is an implication operator.

Proof. Straight.

Recall that an overlap function $G_{O}$ is a particular instance of aggregation function such that $G_{O}(0,1)=G_{O}(1,0)=0$. So we could start just writing a corollary of Proposition 7. However, we can provide a slightly more general result.

Proposition 8. If $G_{O}:[0,1]^{2} \rightarrow[0,1]$ is an overlap function and $N_{1}$ and $N_{2}$ are strong negations, then $I(x, y)=N_{1}\left(G_{O}\left(x, N_{2}(y)\right)\right)$ is a continuous implication operator.

Proof. Straight.

Remark 2. The restriction of $N_{1}$ and $N_{2}$ being strong negations can be relaxed, and the same result holds if we demand that both are only strict (and hence continuous), and even if $N_{1}$ and $N_{2}$ are just continuous negations.

Even if they are negations, without any other additional requirement, we recover an implication operator, although not necessarily continuous.

In any case, involution has an impact in the subsequent examples.

Taking into account the already known methods for constructing overlap functions in terms of automorphisms, as well as Trillas' characterization theorem of strong negations, we can state the following.

Example 5. If $\varphi, \varphi_{1}, \varphi_{2}:[0,1] \rightarrow[0,1]$ are automorphisms, and $G_{O}(x, y)=\varphi^{-1}(\varphi(x) \varphi(y))$ then

$$
I(x, y)=\varphi_{1}^{-1}\left(1-\varphi_{1}\left(\varphi^{-1}\left(\varphi(x) \varphi\left(\varphi_{2}^{-1}\left(1-\varphi_{2}(y)\right)\right)\right)\right)\right)
$$

is an implication operator.

\section{Example 6.}

- Let $\varphi_{1}(x)=\varphi_{2}(x)=\varphi(x)=x$ and $G_{O}(x, y)=$ $\varphi^{-1}(\varphi(x) \varphi(y))$. Then $N_{1}(x)=N_{2}(x)=1-x$ and $G_{O}(x, y)=\varphi^{-1}(\varphi(x) \varphi(y))=x y$. The expression of $I$ is $I(x, y)=N_{1}\left(G_{O}\left(x, N_{2}(y)\right)\right)=N_{1}\left(G_{O}(x, 1-\right.$ $y))=N_{1}(x(1-y))=1-x(1-y)=1-x+x y$. 
- Consider now the automorphisms $\varphi_{1}(x)=x^{2}$, $\varphi(x)=\varphi_{2}(x)=x$, and the overlap function $G_{O}(x, y)=\varphi^{-1}(\varphi(x) \varphi(y))$. Then $N_{1}(x)=(1-$ $\left.x^{2}\right)^{1 / 2}, N_{2}(x)=1-x$ and $G_{O}(x, y)=x y$. The expression of $I$ is $I(x, y)=N_{1}\left(G_{O}\left(x, N_{2}(y)\right)\right)=$ $N_{1}\left(G_{O}(x, 1-y)\right)=N_{1}(x(1-y))=\left(1-x^{2}(1-\right.$ $\left.y)^{2}\right)^{1 / 2}$.

Proposition 9. If $N_{1}, N_{2}$ are strong negations and for $i=1, \ldots, n, G_{O, i}(x, y)$ are overlap functions and $w_{i} \in[0,1]$ are such that $\sum_{i=1}^{n} w_{i}=1$, then

$$
I(x, y)=N_{1}\left(\sum_{i=1}^{n}\left(w_{i} G_{O, i}\left(x, N_{2}(y)\right)\right)\right)
$$

is an implication operator.

Proof. $\mathrm{In}^{18}$ it was proven that the convex combination of overlap function is also an overlap function so the result is straight by Proposition 8 .

Remark 3. Notice that the previous theorem does not hold for every class of aggregation functions, an example being the t-norms, which convex combination does not necessarily yield another t-norm.

From the construction method of overlap functions by means of rational functions ${ }^{10}$ we can also state the following example.

Example 7. If $N_{1}$ and $N_{2}$ are strong negations and

$$
G_{O}(x, y)=\frac{f(x, y)}{f(x, y)+h(x, y)}
$$

for some $f, h:[0,1]^{2} \rightarrow[0,1]$ such that

1. $f$ and $h$ are symmetric;

2. $f$ is non decreasing and $h$ is non increasing;

3. $f(x, y)=0$ if and only if $x y=0$;

4. $h(x, y)=0$ if and only if $x y=1$;

5. $f$ and $h$ are continuous;

then

$$
I(x, y)=N_{1}\left(\frac{f\left(x, N_{2}(y)\right)}{f\left(x, N_{2}(y)\right)+h\left(x, N_{2}(y)\right)}\right)
$$

is an implication operator.
Remark 4. As in Remark 2 strong negations are not required, since the result holds for strict negations.

Example 8. If $f(x, y)=(x y)^{\alpha}, h(x, y)=1-(x y)^{\alpha}$ and $N(x)=N_{1}(x)=N_{2}(x)=\left(1-x^{w}\right)^{1 / w}$ for $\alpha, w>$ 0 , then eq. (1) yields the following implication operator

$$
I(x, y)=\left(1-x^{\alpha w}\left(1-y^{w}\right)^{\alpha}\right)^{1 / w} .
$$

In this situation $I$ does not necessarily verify the properties of Definition 9.

In the remainder of this section we study the restrictions to be imposed to overlaps in order Proposition 8 to yield implications fulfilling specific properties.

Corollary 10. Let $N_{1}$ and $N_{2}$ be strong negations and let $G_{O}$ be an overlap function. Then, if $I(x, y)=N_{1}\left(G_{O}\left(x, N_{2}(y)\right)\right.$, the following statements hold:

1 I satisfies $(S N)$ with $N_{1}$ if and only if $G_{O}(x, 1)=x$ for all $x \in[0,1]$.

$2 I\left(x, N_{2}(x)\right)=N_{1}(x)$ if and only if $G_{O}(x, x)=x$ for all $x \in[0,1]$.

Proof. Straight.

Proposition 11. Let $N_{1}$ and $N_{2}$ be strong negations and let $G_{O}$ be an overlap function. Then, $I(x, y)=N_{1}\left(G_{O}\left(x, N_{2}(y)\right)\right.$ does not satisfy neither $(O P)$ nor $(I P)$ with respect to $N_{2}$.

Proof. As $x \leqslant N_{2}(x)$ or $x>N_{2}(x)$ for all $x \in[0,1]$, if $I$ verifies the property $(O P)$ then:

$$
\begin{gathered}
I\left(x, N_{2}(x)\right)=1=N_{1}\left(G_{O}(x, x)\right) \\
\text { if and only if } G_{O}(x, x)=0
\end{gathered}
$$

or

$$
I\left(N_{2}(x), x\right)=N_{1}\left(G_{O}\left(N_{2}(x), N_{2}(x)\right)\right)=1
$$

if and only if $G_{O}\left(N_{2}(x), N_{2}(x)\right)=0$.

But this is a contradiction with the fact that $G_{O}(x, y)=0$ if and only if $x y=0$.

If $I$ verifies the property $(I P)$ then $1=I(x, x)=$ $N_{1}\left(G_{O}\left(x, N_{2}(x)\right)\right)$, hence $G_{O}\left(x, N_{2}(x)\right)=0$ for all $x \in[0,1]$. But this is also a contradiction with $G_{O}(x, y)=0$ if and only if $x y=0$. 
The following result ${ }^{10}$ characterizes associative overlap functions.

Proposition 12. ${ }^{10}$ If $G_{O}$ is an overlap function satisfying

$$
G_{O}\left(x, G_{O}(y, z)\right)=G_{O}\left(y, G_{O}(x, z)\right)
$$

for all $x, y, z \in[0,1]$, then the following items hold.

1. $G_{O}$ is associative;

2. If $G_{O}$ has neutral element 1 , then

3. If $G_{O}$ has neutral element 1 , then $G_{O}$ is a continuous t-norm.

Theorem 13. If $N$ is a strong negation, $G_{O}(x, y)$ is an overlap function and

$$
I(x, y)=N\left(G_{O}(x, N(y)),\right.
$$

then:

1. I $(x, y)$ satisfies $(C P)$.

2. $I(x, y)$ is a continuous function; namely, (IB) is satisfied.

\section{Proof. Straight.}

In the following example we provide counterexamples for the properties of Definition 9 to implication operators which are built from overlap functions.

Example 9. Consider the family of overlap functions $G_{O}(x, y)=(x y)^{p}$ for $p>0$. From Eq. (2), let $I_{p}$ be the implication operator constructed through $G_{O}$ with exponent $p$ using $N(x)=1-x$.

1. If $p=2$, then $I_{2}(1, x)=1-1^{2}(1-x)^{2}=2 x-$ $x^{2} \neq x$, so $I_{2}$ does not satisfy property $(N P)$.

2. If $p=2$, then

$$
\begin{aligned}
& I_{2}(1 / 2, I(1 / 3,1 / 4))=I_{2}(1 / 2,15 / 16) \\
& =1023 / 1024 \neq I_{2}\left(1 / 3, I_{2}(1 / 2,1 / 4)\right) \\
& =I_{2}(1 / 3,55 / 64)=4087 / 4096,
\end{aligned}
$$

so $I_{2}$ does not satisfy $(E P)$.
3. If $p=1$, then $I_{1}(x, x)=1-x(1-x)=1-x+$ $x^{2} \neq 1$ for all $\left.x \in\right] 0,1\left[\right.$, so $I_{1}$ does not satisfy $(I P)$.

4. If $p=1 I_{1}(1 / 3,1 / 2)=1-1 / 3 \cdot 1 / 2=1-$ $1 / 6=5 / 6 \neq 1$, so $I_{1}$ does not satisfy $(O P)$.

5. If $p=2$, then $N_{I}(x)=I_{2}(x, 0)=1-x^{2}$. Hence $N_{I}$ is a negation, but it is not a strong negation because $N_{I}\left(N_{I}(x)\right)=N_{I}\left(1-x^{2}\right)=1-(1-$ $\left.x^{2}\right)^{2} \neq x$. So $I_{2}$ does not satisfy $(S N)$.

6. Let $p=\frac{1}{3}, I_{\frac{1}{3}}(1,7 / 8)=1-(1 \cdot 1 / 8)^{1 / 3}=$ $1-1 / 2=1 / 2<7 / 8$, so $I_{\frac{1}{3}}$ does not satisfy (IA).

Example 10. Let be $I$ an implication operator constructed as eq. (2). Then:

1. If $G_{O}(x, y)=(x y)^{p}$, for $p>0$ and

$$
N(x)=\frac{1-x}{1+\lambda x}
$$

with $\lambda>-1$ then

$$
I(x, y)=\frac{1-\left(\frac{x-x y}{1+\lambda y}\right)^{p}}{1+\lambda\left(\frac{x-x y}{1+\lambda y}\right)^{p}} .
$$

This implication function does not necessarily verify the properties of Definition 9.

2. If $G(x, y)=\frac{2 x y}{x+y}$, then

$$
I(x, y)=N\left(\frac{2 x N(y)}{x+N(y)}\right) .
$$

verifies the property $(I C)$ because

$$
I(x, N(x))=N(G(x, x))=N(x) .
$$

However, it does not necessarily verify any other property of Definition 9. 


\section{Conclusions}

In this work we have studied the construction of negations and implication operators through a particular class of aggregation functions, namely, overlap functions. The analysis that we have carried out can be extended to the dual of overlap functions, grouping functions, and most of the results will just need a rephrasing.

However, this is only the first step of a more ambitious program. Since overlap and grouping functions can provide, in some situations (where associativity is not required), a replacement of t-norms and t-conorms, it seems natural to develop an extensive study on both residual implications and (S,N)implications defined in terms of overlap and grouping functions, with an eye kept on possible applications, specially in the field of image processing.

\section{Acknowledgement}

This research has been partially supported by Grant TIN2013-40765-P from the Government of Spain and the Research Services of the Universidad Publica de Navarra.

\section{References}

1. M.Baczyński, and B.Jayaram, Fuzzy Implications, Studies in Fuzziness and Soft Computing, 231 Springer, Berlin, 2008.

2. M.Baczyński, G. Beliakov, H. Bustince, and A. Pradera (Eds.), Advances in Fuzzy Implication Functions, Studies in Fuzziness and Soft Computing, 300 Springer, Berlin, 2013.

3. W. Bandler, and L. Kohout, Fuzzy power sets and fuzzy implication operators, Fuzzy Sets and Systems, 4 (1) (1980), 13-30.

4. H. Bustince, P. Burillo and F. Soria, Automorphisms, negations and implication operators, Fuzzy Sets and Systems, 134 (2003), 209-229.

5. H. Bustince, E. Barrenechea and M. Pagola, Restricted equivalence functions, Fuzzy Sets and Systems, 157 (2006), 2333-2346.

6. H. Bustince, M. Pagola, and E. Barrenechea, Construction of fuzzy indices from fuzzy DI-subsethood measures: Application to the global comparison of images, Information Sciences, 177 (3) (2007), 906-929.
7. H. Bustince, E. Barrenechea, and M. Pagola, Image thresholding using restricted equivalence functions and maximizing the measures of similarity, Fuzzy Sets and Systems, 158 (5) (2007), 496-516.

8. H. Bustince, E. Barrenechea, and M. Pagola, Relationship between restricted dissimilarity functions, restricted equivalence functions and normal EN-functions: Image thresholding invariant, Pattern Recognition Letters, 29 (4) (2008), 525-536.

9. H. Bustince, B. De Baets, J. Fernandez, R. Mesiar and J. Montero, A generalization of the migrativity property of aggregation functions, Information Sciences, 191 (2012), 76-85.

10. H. Bustince, J. Fernandez, R. Mesiar, J. Montero and R. Orduna, Overlap functions, Nonlinear Analysis 72 (2010), 1488-1499.

11. H. Bustince, J. Montero and R. Mesiar, Migrativity of aggregation functions, Fuzzy Sets and Systems, 160 (2009), 766-777.

12. H. Bustince, J. Fernandez, J. Sanz, M. Baczyński, and R. Mesiar, Construction of strong equality index from implication operators, Fuzzy Sets and Systems, 211 (16) (2013), 15-33.

13. H. Bustince, M. Pagola, R. Mesiar, E. Hüllermeier, and F. Herrera, Grouping, overlap, and generalized bientropic functions for fuzzy modeling of pairwise comparisons, IEEE Transactions on Fuzzy Systems, 20 (3) (2012), 405-415.

14. K. Demirli, and B. De Baets, Basic properties of implicators in a residual framework, Tatra Mountains Mathematical Publications 16 (1999), 31-46.

15. J. Dombi, Basic concepts for a theory of evaluation: the aggregative operator, European Journal of Operations Research 10 (1982), 282-293.

16. J. Fodor and M. Roubens, Fuzzy Preference Modelling and Multicriteria Decision Support, in: Theory and Decision Library (Kluwer Academic Publishers) 1994.

17. M. Grabisch, J.-L. Marichal, R. Mesiar and E. Pap. Aggregation Functions. Cambridge University Press, Cambridge, (2009).

18. A. Jurio, H. Bustince, M. Pagola, A. Pradera, and R.R. Yager, Some properties of overlap and grouping functions and their application to image thresholding, Fuzzy Sets and Systems 229 (2013), 69-90.

19. G.J. Klir and T.A. Folger, Fuzzy Sets, Uncertainty and Information, Prentice Hall, Englewood Cliffs, NJ, (1988).

20. M. Mas, M. Monserrat, J. Torrens, and E. Trillas, A survey on fuzzy implication functions. IEEE Transactions on Fuzzy Systems 15(6)(2007) 1107-1121

21. S.V. Ovchinnikov, and M. Roubens, On strict preference relations, Fuzzy Sets and Systems, 43 (1991), 319-326.

22. B. Schweizer, and A. Sklar, Probabilistic Metric 
Spaces, North-Holland, Amsterdam (1983).

23. E.Trillas, Sobre funciones de negación en la teoría de conjuntos difusos, Stochastica III(1), 47-60, 1979[English translation in: S.Barro, A. Bugarin, A.Sobrino (Eds.), Advances in Fuzzy Logic, Public. Univ. Santiago de Compostela, Spain, 1998, 31-45,].

24. E.Trillas, and L. Valverde, On some functionally expressable implications for fuzzy set theory. Proc. of the 3rd Inter. Seminar on Fuzzy Sets Theory, Linz, Austria, (1981), 173-190.

25. R. Yager, On some new classes of implication operators and their role in approximate reasoning, Information Sciences, 167 (2004), 193-216.

26. L. A. Zadeh, Fuzzy sets as a basis for a theory of possibility, Fuzzy Sets and Systems, 1, 1978,3-28. 